\section{A) Check for updates}

Cite this: Food Funct., 2019, 10, 5739

\title{
Shape up! How shape, size and addition of condiments influence eating behavior towards vegetables
}

\author{
Arianne van Eck, (D) ${ }^{\text {a,b }}$ Christien Wijne, ${ }^{c}$ Vincenzo Fogliano, ${ }^{\text {a,b }}$ Markus Stieger $^{\mathrm{a}, \mathrm{b}}$ and \\ Elke Scholten*a,c
}

\begin{abstract}
Practical approaches to increase consumption of healthy foods such as vegetables are needed. Controlling eating rate is a promising strategy, since faster eating rates have been related to higher food intake. Food properties can be modified to influence eating rates, but little is known about the impact of vegetable dimensions and condiment additions on eating rates of vegetables. This study determined the influence of shape, size and condiment properties on eating behavior towards carrots. Eating behavior (mastication time, number of chews, chewing frequency, eating rate) was determined for carrots with same total weight but different shapes (cube, julienne), and varying in size, number of pieces and aspect ratio. Carrots presented in one large cube required the lowest mastication effort (shortest mastication time, fewest chews) among all pre-cut carrots. Carrot cubes required less mastication effort leading to higher eating rates than carrots julienne. To investigate the effect of condiment addition on eating behavior towards carrots, mayonnaises varying in fat content and viscosity were combined with carrots, and mastication behavior and bolus properties were determined. Mayonnaises, in particular those with high fat content or low viscosity, contributed to faster bolus formation of carrots. Carrots were swallowed with less particles of larger sizes when mayonnaises were added. These results indicate that a specific particle size is not a prerequisite to induce swallowing, and that other bolus properties such as lubrication or cohesiveness trigger the urge to swallow. We conclude that eating behavior towards carrots can be controlled by relatively small changes in both carrot and condiment properties. To increase carrot intake by increasing eating rate, we suggest to avoid cutting of carrots or to add condiments, which could be an effective strategy to increase vegetable consumption or to decrease mastication effort to target the elderly population.
\end{abstract}

Received 6th June 2019, Accepted 18th August 2019 DOI: $10.1039 /$ c9fo01206k rsc.li/food-function
2012-2014, and only $15 \%$ of Dutch adults met the recommended amount of $200 \mathrm{~g}$ day $^{-1}$. $^{5,6}$ Practical approaches to increase the consumption of vegetables are needed, and therefore a better understanding of the factors that influence vegetable intake is necessary.

One of the approaches to increase vegetable intake is by controlling eating rate. ${ }^{7}$ Eating rate $\left(\mathrm{g} \mathrm{min}^{-1}\right)$ directly relates to oro-sensory exposure time, which is a main determinant of satiation and ad libitum intake. Faster eating rates have been related to higher food intake, and increasing the eating rates of vegetables is therefore desired to lower satiation and increase vegetable intake. Eating rates are known to depend on both consumer characteristics and food properties. ${ }^{8,9,10-12}$ Unfortunately, consumer characteristics such as age, gender and ethnicity are fixed and cannot be influenced. Food properties such as food texture and shape, on the other hand, can be modified, which can be used as an effective strategy to influence eating rate, satiation and intake of vegetables.

\footnotetext{
${ }^{a}$ TiFN, P.O. Box 557, 6700 AN Wageningen, The Netherlands.

E-mail:elke.scholten@wur.nl

${ }^{b}$ Food Quality and Design, Wageningen University, P.O. Box 17, $6700 \mathrm{AA}$

Wageningen, The Netherlands

${ }^{c}$ Physics and Physical Chemistry of Foods, Wageningen University, P.O. Box 17, 6700 AA Wageningen, The Netherlands
} 
Vegetables naturally differ in shape and size, and consumers use multiple preparation techniques such as cutting, cooking, steaming and baking before vegetable consumption. These preparation techniques often soften the vegetables. It is well-known that softer foods require lower mastication efforts (mastication time, number of chews, muscle activity), are consumed at higher eating rates leading to higher intake, than harder foods. ${ }^{13-16}$ However, less is known about the effect of shape and size of vegetables on eating behavior and ad libitum intake. Kohyama et al. (2007) investigated eating behavior towards carrot cubes $(20 \times 20 \times 20 \mathrm{~mm}, \sim 7 \mathrm{~g})$ and finely cut carrots (fine strips of $1 \times 1.5 \times 30 \mathrm{~mm}, \sim 7 \mathrm{~g}$ ). Finely cut carrots required longer mastication time, more chews with a higher muscle activity, and were consumed at lower eating rates than carrot cubes. ${ }^{17}$ However, it is not well understood why there is a difference in eating behavior between carrot cubes and carrots julienne. The effect of carrot shape on bolus formation was not determined in this study. Investigating the bolus properties of carrots varying in shape might help to explain the observed differences in eating behavior. Recently, Goh et al. (2017) and Liem et al. (2019) investigated the influence of vegetable size on vegetable intake. Intake of whole carrots $(160 \times$ $30 \times 20 \mathrm{~mm}, \sim 70 \mathrm{~g})$ and carrot cubes $(20 \times 20 \times 20 \mathrm{~mm}, \sim 10 \mathrm{~g})$ by children was determined in a controlled school setting and a real-life movie setting. Intake of whole carrots was higher than intake of carrot cubes in both settings (consumption increased with $75 \%$ in the movie setting), demonstrating that carrot shape influences carrot intake. ${ }^{18,19}$ However, it is not clearly understood how the size of vegetables influences eating behavior and bolus formation. Taking the mastication behavior and bolus properties of vegetables into consideration allows to better understand the mechanisms underlying vegetable intake. Based on this knowledge, practical approaches to increase the consumption of vegetables might be devised.

Vegetables are frequently consumed together with condiments, for example with dips (vegetables cut in beams, combined with mayonnaise, hummus or yogurt-based dressing), as Russian salad (vegetables cooked and cut in small cubes, combined with mayonnaise or salad dressings) or coleslaw (vegetables cut julienne, combined with mayonnaise or salad dressing). The development of fat-reduced condiments has raised over the past years, to provide flavors to meals without adding high levels of fat or calories. However, little is known about the effect of condiment addition and its properties on eating behavior towards vegetables. In the case of carrier foods with relatively low water content such as toast or crackers, it is known that condiments facilitate bolus formation by moistening and softening food boli, which leads to shorter mastication times to break down and lubricate boli enough to be safely swallowed. $^{20-22}$ The shorter mastication times are due to the ability of toast and crackers to absorb moisture from saliva or condiments, which softens these foods by increasing moisture content. $^{22}$ Vegetables are quite different from toast and crackers, as they have a high moisture content and are speculated to not absorb moisture from saliva or condiments during mastication. Therefore, vegetables require different oral processing strategies than toast and crackers. ${ }^{23,24}$ The impact of condiments on bolus formation is expected to be different for vegetables than for toast or crackers. Instead of acting as a food softener, the condiments will just be mixed with the vegetables. Further understanding of the effect of condiment properties on mastication behavior towards vegetables such as carrots may be used to control food intake of different vegetable-condiment combinations.

The objective of this study was to determine the effect of shape, size and addition of condiments differing in physicochemical properties on eating behavior towards carrots. We chose mayonnaise as a representative for the condiment, as it is often used with carrots to make salads or used as an ingredient for dips. Raw carrots were cut into cubes and julienne pieces of various sizes and aspect ratios, which were assessed with and without mayonnaise. Carrots cut into cubes or julienne represent simplified model foods for vegetables with dip and vegetable salads such as coleslaw. We hypothesize that carrots varying in shape are consumed with different mastication efforts and eating rates leading to different bolus properties at the moment of swallowing. The addition of mayonnaise to carrots is hypothesized to stick carrot pieces together by which it aids the bolus formation of carrots. Studying the effect of vegetable shape and size and the addition of condiments on eating behavior towards vegetables is relevant because it imparts structural changes during consumption, thereby impacting bioaccessibility of ingredients, oro-sensory exposure time, satiation and food intake. This knowledge can be used to alter the eating behavior of specific target consumer groups, such as the increasing elderly population.

\section{Materials and methods}

\subsection{Carrot preparation}

An overview of all carrot samples is presented in Table 1. Fresh raw carrots (Daucus carota; winter carrot; purchased from local retailer in Wageningen, Netherlands) were peeled and cut into cubes $(15 \times 15 \times 15 \mathrm{~mm})$ and julienne pieces $(90 \times 3 \times 2 \mathrm{~mm})$, which were further cut to obtain cube and julienne samples varying in number and size of carrot pieces. All samples were served at similar total weight of approximately $4.2 \mathrm{~g}$. For the carrot cubes, carrots were cut into one large cube, eight medium-sized cubes, 27 small-sized cubes and 64 very smallsized cubes using a knife. The sample codes used for the cubes are C_1, C_8, C_27 and C_64, in which the first letter refers to the shape (Cube $\mathrm{C}$ ), and the number refers to the number of pieces. For the julienne carrots, carrots were cut using a spiral slicer (Gefu Spiral Slicer Spirelli 2.0) and a knife into eight long julienne pieces, 24 medium-long julienne pieces and 64 short julienne pieces. The sample codes used for the carrot julienne are J_8, J_24 and J_64, in which the first letter refers to the shape (Julienne J), and the number refers to the number of pieces. All carrot samples were prepared freshly the morning of the session and vacuum packed until serving to minimize water loss. 
Table 1 Overview of carrot samples varying in shape, number and size

\begin{tabular}{|c|c|c|c|c|c|c|c|}
\hline Sample code & C_1 & C_8 & C_27 & C_64 & J_8 & J_24 & J_64 \\
\hline \multicolumn{8}{|l|}{ Carrot images } \\
\hline Shape & Cube & Cubes & Cubes & Cubes & Julienne & Julienne & Julienne \\
\hline Number of pieces & 1 & 8 & 27 & 64 & 8 & 24 & 64 \\
\hline Size of pieces & Large & Medium & Small & Very small & Long & Medium & Short \\
\hline Length of one piece (mm) & 15 & 7.5 & 5 & 3.75 & 90 & 30 & 11 \\
\hline Width of one piece (mm) & 15 & 7.5 & 5 & 3.75 & 3 & 3 & 3 \\
\hline Height of one piece (mm) & 15 & 7.5 & 5 & 3.75 & 2 & 2 & 2 \\
\hline Aspect ratio of one piece ${ }^{a}$ & 1 & 1 & 1 & 1 & 30 & 10 & 4 \\
\hline Surface area of one piece $\left(\mathrm{mm}^{2}\right)$ & 1350 & 338 & 150 & 84 & 912 & 312 & 125 \\
\hline Total surface area of all pieces $\left(\mathrm{mm}^{2}\right)$ & 1350 & 2700 & 4050 & 5400 & 7296 & 7488 & 7968 \\
\hline Total weight of all pieces $(\mathrm{g})$ & 4.2 & 4.2 & 4.2 & 4.2 & 4.2 & 4.2 & 4.2 \\
\hline
\end{tabular}

${ }^{a}$ Aspect ratio is here defined as the length of one piece divided by the width of one piece.

\subsection{Mayonnaise preparation}

The mayonnaise formulations and properties are summarized in Table 2. Mayonnaises were prepared using sunflower oil, egg yolk, lemon juice and mustard. All ingredients were purchased from a local retailer in Wageningen, the Netherlands. Three mayonnaises (full fat/high viscosity, low fat/high viscosity, low fat/low viscosity) varying in fat content (77 and $28 \%$ $\mathrm{w} / \mathrm{w}$ ) and viscosity (high viscosity: approximately $190 \mathrm{~Pa}$ s at $1 \mathrm{~s}^{-1}$; low viscosity: approximately $21 \mathrm{~Pa} \mathrm{~s}$ at $1 \mathrm{~s}^{-1}$ ) were prepared. The sample codes used for the mayonnaises are FF_HV, LF_HV, and LF_LV, in which the first two letters refer to full fat (FF) or low fat (LF), and the last two letters to high viscosity (HV) or low viscosity (LV). Mayonnaises were prepared at room temperature $\left(20 \pm 1^{\circ} \mathrm{C}\right)$ using a mixer (Thermomix, Vorwerk, Germany, speed 3). For the FF_HV mayonnaise, $16 \mathrm{~g}$ egg yolk, $3 \mathrm{~g}$ lemon juice and $5 \mathrm{~g}$ mustard were mixed and stirred for 10 $\mathrm{s}$, after which $80 \mathrm{~g}$ sunflower oil was gradually added in $3 \mathrm{~min}$.
For the low-fat mayonnaises (LF_HV and LF_LV), first xanthan (5 $\mathrm{g}$ for LF_HV and $2 \mathrm{~g}$ for LF_LV) (E415, Pit\&Pit bvba, Belgium) was gradually added to $50 \mathrm{~g}$ water under continuous stirring, and the mixture was heated at $80{ }^{\circ} \mathrm{C}$ for 5 minutes until all xanthan was dissolved. The solution was cooled down to room temperature, and then, $16 \mathrm{~g}$ egg yolk, $3 \mathrm{~g}$ lemon juice and $5 \mathrm{~g}$ mustard were added and mixed for $10 \mathrm{~s} .30 \mathrm{~g}$ sunflower oil was then added slowly in $3 \mathrm{~min}$. All mayonnaises were mixed for 2 min after addition of the oil.

To characterize the flow properties of the three mayonnaises, flow curves were determined using a rheometer (Anton Paar Rheometer 301) equipped with an Inset I-PP50/SS plate and a CP50-1 cone. The viscosity of the mayonnaises was measured by putting $0.5 \mathrm{~g}$ mayonnaise on the plate, applying a resting period of $5 \mathrm{~min}$, and then shearing at shear rates ranging from $1 \mathrm{~s}^{-1}$ to $1000 \mathrm{~s}^{-1}$ in $5 \mathrm{~min}$ (30 measurement points of $10 \mathrm{~s}$ each). Measurements were performed for each new batch $(n=5)$ in duplicate at room temperature $\left(20 \pm 1^{\circ} \mathrm{C}\right)$.

Table 2 (A) Formulations of mayonnaises and relative concentrations of all ingredients $(\% \mathrm{w} / \mathrm{w})$ and (B) properties of mayonnaises varying in fat content and viscosity (mean $\pm \mathrm{SD}$ )

\begin{tabular}{|c|c|c|c|c|c|c|}
\hline \multirow[b]{2}{*}{ (A) Formulation } & \multicolumn{2}{|c|}{ Full fat/high viscosity (FF_HV) } & \multicolumn{2}{|c|}{ Low fat/high viscosity (LF_HV) } & \multicolumn{2}{|c|}{ Low fat/low viscosity (LF_LV) } \\
\hline & Mass $(\mathrm{g})$ & Concentration $(\% \mathrm{w} / \mathrm{w})$ & Mass (g) & Concentration $(\% \mathrm{w} / \mathrm{w})$ & Mass (g) & Concentration $(\% \mathrm{w} / \mathrm{w})$ \\
\hline Sunflower oil & 80 & 77 & 30 & 28 & 30 & 28 \\
\hline Egg yolk & 16 & 15 & 16 & 15 & 16 & 15 \\
\hline Lemon juice & 3 & 3 & 3 & 3 & 3 & 3 \\
\hline Mustard & 5 & 5 & 5 & 5 & 5 & 5 \\
\hline Xanthan & - & - & 5 & 5 & 2 & 2 \\
\hline Water & - & - & 50 & 46 & 50 & 47 \\
\hline \multicolumn{2}{|c|}{ (B) Mayonnaise properties } & \multicolumn{2}{|c|}{ Full fat/high viscosity (FF_HV) } & Low fat/high viscosity (LF_HV) & \multicolumn{2}{|c|}{ Low fat/low viscosity (LF_LV) } \\
\hline \multicolumn{2}{|c|}{ Fat content $(\% \mathrm{w} / \mathrm{w})$} & \multicolumn{2}{|l|}{77} & 28 & \multicolumn{2}{|c|}{28} \\
\hline \multirow{2}{*}{\multicolumn{2}{|c|}{$\begin{array}{l}\text { Viscosity at } 1 \mathrm{~s}^{-1}(\mathrm{~Pa} \mathrm{~s}) \\
\text { Viscosity at } 10 \mathrm{~s}^{-1}(\mathrm{~Pa} \mathrm{~s})\end{array}$}} & \multicolumn{2}{|l|}{$186 \pm 79$} & $193 \pm 37$ & \multicolumn{2}{|c|}{$21 \pm 5$} \\
\hline & & \multirow{2}{*}{\multicolumn{2}{|c|}{$\begin{array}{l}29 \pm 8 \\
5+1\end{array}$}} & $15 \pm 3$ & \multirow{2}{*}{\multicolumn{2}{|c|}{$\begin{array}{l}4 \pm 1 \\
1 \pm 0\end{array}$}} \\
\hline \multicolumn{2}{|c|}{ Viscosity at $100 \mathrm{~s}^{-1}(\mathrm{~Pa} \mathrm{~s})$} & & & $2 \pm 1$ & & \\
\hline
\end{tabular}




\subsection{Carrots varying in shape}

The mastication behavior towards carrots varying in shape, number of pieces and size of pieces (Table 1) was determined. 17 subjects (7 male and 10 female, between 20 and 34 years old) participated. All subjects were European, Caucasian healthy adults with good dental health (self-reported), nonsmoking habits (self-reported) and frequent carrot consumers (self-reported). Each subject attended one session of $45 \mathrm{~min}$ during which mastication behavior was determined using video recordings (section 2.5). The mastication behavior towards four carrot cubes (C_1, C_8, C_27 and C_64) and three julienne carrots (J_8, J_24 and J_64) was quantified. This yielded a total of 7 samples, which were assessed in duplicate. All samples were served at a weight of $4.2 \mathrm{~g}$. Samples were served on a spoon in randomized order following a completely randomized design. Samples were coded with 3 digit codes. Subjects cleaned their palate after consumption of each sample with water and crackers. Subjects gave written informed consent and received financial compensation for participation.

\subsection{Carrots without and with mayonnaises varying in fat content and viscosity}

The mastication behavior and bolus properties of carrots differing in shape without and with mayonnaises varying in fat content and viscosity (FF_HV, LF_HV and LF_LV) were determined. Carrot cubes (C_1) and carrots julienne (J_8) were used, since these carrots were the most different with respect to mastication behavior (see section 3.1). 20 subjects (9 male and 11 female, between 18 and 25 years old) participated. All subjects were European, Caucasian healthy adults with good dental health (self-reported), non-smoking habits (selfreported), frequent carrot consumers (self-reported), and no allergies or intolerances for mayonnaises. Seven of the subjects also participated in the previous test (carrots varying in shape), whereas 13 participants performed only this one. Each subject attended three sessions of 60 min over a time period of four weeks. Mastication behavior was determined in duplicate in the first session (section 2.5). Bolus properties (particle size distribution, retention of carrot in the bolus) were determined in the following two sessions (section 2.6). Carrots (C_1, J_8) without and with mayonnaises (FF_HV, LF_HV, LF_LV) were assessed, which gave a total of 8 samples. Carrots were served at approximately $4.2 \mathrm{~g}$ and carrots with added mayonnaises at approximately $6.2 \mathrm{~g}$ (4.2 g carrot with $2.0 \mathrm{~g}$ mayonnaise). This carrot/mayonnaise weight ratio corresponds to a common vegetable/mayonnaise serving ratio used to prepare coleslaw. For carrot cubes, mayonnaise was added on top of the cubes. For carrots julienne, carrots were premixed with the mayonnaises in a plastic container before serving. Samples were served on a spoon in randomized order following a completely randomized design. Samples were coded with 3 digit codes. Subjects cleaned their palate after consumption of each sample with water and crackers. Subjects gave written informed consent and received financial compensation for participation.

\subsection{Characterization of mastication behavior using video recordings}

Mastication behavior (total mastication time, number of chews, chewing frequency and eating rate) was characterized in duplicate using video recordings, as described by AguayoMendoza et al. (2019) and van Eck et al. (2019). ${ }^{13,22}$ Subjects were seated in a chair with a video camera placed in front of them at a distance of approximately $50 \mathrm{~cm}$. At the start of the session, the researcher placed two stickers with a distance of $5 \mathrm{~cm}$ on the subjects' forehead, one sticker on the subjects' nose tip, one sticker on the subjects' chin, and one sticker on the subjects' Adam's apple. These stickers were used as reference points during the video analyses. Subjects were instructed to chew each sample as they would do naturally, to maintain their head straight to the camera, not to block their face with their hand, and to raise their hand to indicate the moment of swallowing.

Video analyses were performed using Kinovea software (version 0.8.15) to determine total mastication time (s), number of chews and chewing frequency (chews per s). Each video was calibrated by setting the distance between the two stickers on the forehead as $5 \mathrm{~cm}$. The $Y$ coordinates of the nose and chin stickers were extracted over time. All analyses were performed at 15 frames per second.

Total mastication time ( $\mathrm{s}$ ) was defined as the time period from placing the sample in the mouth (i.e. the moment the lips were closed after placing the food in the mouth) until the moment of swallowing (i.e. the moment that the subjects raised their hand to indicate swallowing, verified by a movement of the Adam's apple sticker). Subjects generally took multiple swallows during mastication, and only the main swallow (i.e. the moment they swallowed the bolus) was used to determine total mastication time. The number of chews was determined by tracking the difference in $Y$ coordinates between the nose and the chin sticker (i.e. vertical displacement) during total mastication time. Chewing frequency was calculated by dividing the number of chews by the mastication time. Eating rate $\left(\mathrm{g} \mathrm{min}^{-1}\right)$ was calculated by dividing the sample weight by the total mastication time in minutes.

\subsection{Characterization of bolus properties}

Subjects were instructed to chew the samples and to expectorate the bolus at the moment of swallowing (i.e. 100\% of total mastication time). This time point was determined for each sample by averaging the mastication times of all subjects and all replicates obtained during the video recordings. Boli were collected in pre-weighed containers covered with lids to prevent moisture evaporation from the samples. Immediately after bolus expectoration, subjects were instructed to take a sip of water, to thoroughly rinse their mouth and to expectorate the debris in a separate container.

2.6.1 Carrot retention and loss. The containers with expectorated bolus were weighed to determine the weight of the total bolus. After weighing, the bolus was sieved with a $1 \mathrm{~mm}$ sieve with water for $1 \mathrm{~min}$, washed with acetone, and left in 
the sieve for $1 \mathrm{~min}$. This process caused the mayonnaise and/ or saliva to pass through the sieve and the majority of carrot particles to be retained. Hence, carrot particles less than $1 \mathrm{~mm}$ were discarded (typically less than 1\% of all carrot particles). The retained carrot particles were weighed in order to determine the weight of carrot retained in the bolus $\left(m_{\mathrm{b}}\right)$. This approach (i.e. sieving, washing, drying and weighing) was also applied to the debris samples to determine the weight of carrot retained in the debris $\left(m_{\mathrm{d}}\right)$. Mass of lost carrots $\left(m_{\text {lost }}\right)$ due to swallowing was calculated using $m_{\text {lost }}=m_{0}-\left(m_{\mathrm{b}}+m_{\mathrm{d}}\right)$, where $m_{0}$ is the weight of carrot before consumption (i.e. $4.2 \mathrm{~g}$ ). This calculation was based on the assumption that the bolus was fully consumed (typically no carrot pieces were left on the spoon).

2.6.2 Particle size distribution. Following carrot retention and loss, particle size distribution of carrots in the bolus was determined using image analysis. For each bolus, the researcher placed two times $0.5 \mathrm{~g}$ of carrot particles obtained from the bolus in a Petri dish $(120 \times 120 \times 17 \mathrm{~mm})$. Hence, $1 \mathrm{~g}$ of the total bolus ( $0.5 \mathrm{~g}$ in duplicate) was used for particle size characterization. Individual particles were separated from each other using a spatula. The Petri dishes were placed on a flatbed scanner (Canon CanoScan 9000F MarkII) and a color picture with a black background was taken. Pictures were imported into ImageJ (version 1.51f, National Institute of Health, USA) to conduct particle size analysis. Pictures were converted to an 8-bit image, after which a black and white threshold was applied to obtain a binary image. For each image, the number of particles, average $d_{50}$ particle area $\left(\mathrm{mm}^{2}\right)$ and average particle roundness were obtained. Since carrot particles were not spherical, the area rather than diameter was taken as a measure to represent particle size.

\subsection{Data analysis}

Results were reported as mean values with standard error. Outliers $(Z$-score $>3.29)$ were removed from the data (typically less than $1.2 \%$ of all values). To investigate the mastication behavior towards carrots varying in shape, size and number, linear mixed models were performed with shape as fixed effect and subject, replicate and serving order as random effects using Lmer package. ${ }^{25}$ In addition, Pearson's product-moment correlations $(r)$ were used to determine the relationships between carrot shape properties (number of pieces, surface area, aspect ratio) and mastication behavior parameters (mastication time, number of chews, eating rate). To investigate the addition of mayonnaises on mastication behavior and bolus properties of carrots, linear mixed models were performed with mayonnaise, shape and mayonnaise:shape as fixed effects and subject as random effect using Lmer package. ${ }^{25}$ In addition, Principal Component Analysis (PCA) was performed on the bolus properties data, in which the mastication behavior parameters (total mastication time, number of chews) were plotted as supplementary variables using SensorMineR package. ${ }^{26} \mathrm{R}$ language (RStudio, version 1.0.143) was used to perform all statistical tests. Significance level of $p<0.05$ was chosen.

\section{Results and discussion}

\subsection{Influence of shape, number and size of carrot pieces on mastication behavior}

Fig. 1 shows the total mastication time (A), the number of chews until swallowing (B) and the eating rate (C) for the different carrot samples. All samples had similar weight (approximately $4.2 \mathrm{~g}$ ), so the differences can be attributed to a separate effect of the shape, size or number of pieces. Total mastication time $(F=25.5, p<0.001)$, number of chews required to swallow carrots $(F=15.3, p<0.001)$ and eating rate $(F=21.1, p<0.001)$ were significantly influenced by the way carrots were cut before consumption. Even though subjects adapted the time and number of chews to a large extent, their chewing frequencies did not differ between samples varying in
(A)

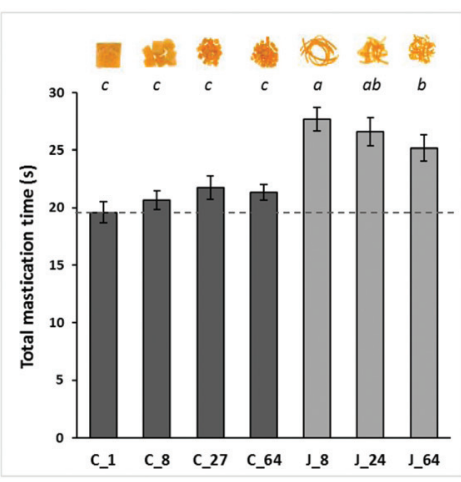

(B)

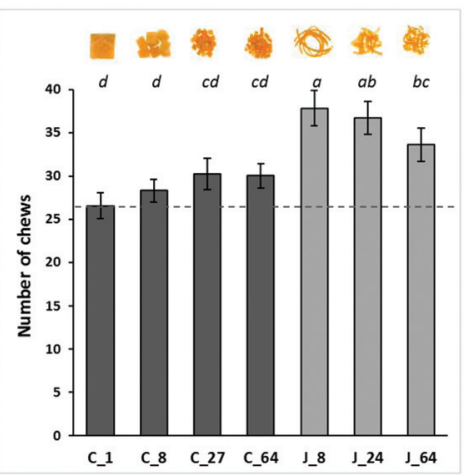

(C)

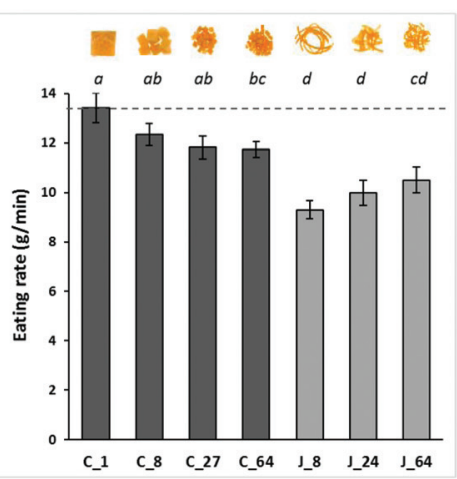

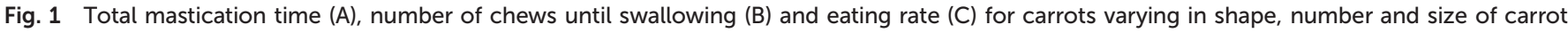

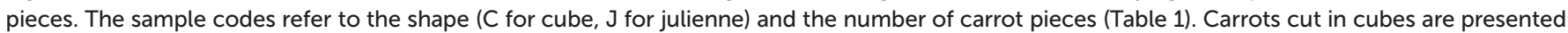

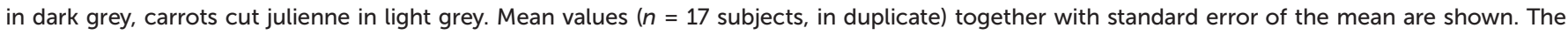

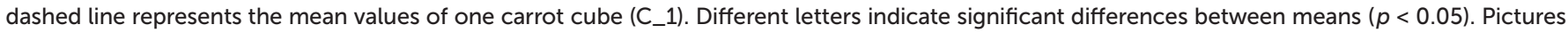
of the carrot samples are presented for illustration purposes. 
the number, size and shape of carrot pieces $(F=0.6, p=$ 0.695). Chewing frequency of all carrot samples was about 1.4 chews per s. This is in line with previous studies on eating behavior towards raw carrots. ${ }^{11,13,17}$

Carrots cut into one cube (C_1) were consumed with the shortest chewing time (20 $\pm 1 \mathrm{~s})$, fewest chews $(27 \pm 2)$ and

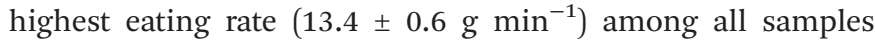
tested. Cutting carrots into multiple smaller cubes (C_8, C_27 and C_64) did not significantly affect mastication times (21 \pm 1 s, $22 \pm 1 \mathrm{~s}$ and $21 \pm 1 \mathrm{~s}$ ), numbers of chews $(28 \pm 1,30 \pm 2$, $30 \pm 1)$ and eating rates $\left(12.3 \pm 0.4 \mathrm{~g} \mathrm{~min}^{-1}, 11.8 \pm 0.5 \mathrm{~g} \mathrm{~min} \mathrm{~m}^{-1}\right.$, $11.7 \pm 0.3 \mathrm{~g} \mathrm{~min}^{-1}$ ) compared to C_1. Much larger effects were observed when carrots were cut into julienne pieces (J_8, J_24 and J_64), leading to increased total mastication times, increased number of chews and decreased eating rates. Consumption of J_8, J_24 and J_64 required $28 \pm 1 \mathrm{~s}$ and $38 \pm$ 2 chews $(p<0.001, p<0.001), 27 \pm 2 \mathrm{~s}$ and $37 \pm 2$ chews $(p<$ $0.001, p<0.001)$ and $25 \pm 1 \mathrm{~s}$ and $34 \pm 2$ chews $(p<0.001, p<$ 0.001 ), respectively. Eating rates of J_8, J_24 and J_64 were 9.3 $\pm 0.4,10.0 \pm 0.5$ and $10.5 \pm 0.5 \mathrm{~g} \mathrm{~min}^{-1}$, respectively. This result indicates that cutting carrots, in particular into julienne shape, increases rather than reduces mastication effort required to form a bolus that is safe to swallow and consequently reduces eating rate. This observation might be explained by the selection function, which describes that larger particles are more likely to be selected by the molars to be further broken-down during chewing than smaller particles. ${ }^{27,28}$ Pre-cut carrots have a higher number of smaller particles already at the beginning of mastication, which leads to a lower particle selection chance and consequently the consumption of smaller carrot pieces requires a higher mastication effort to break down all particles to a certain size to form a bolus that is safe to swallow. Similar findings were reported by Kohyama et al., who found that finely cutting carrots increased consumers mastication effort. ${ }^{17}$ However, for other foods such as gelatin gels, surimi gels or roast pork, no effect of pre-cutting on the mastication effort has been observed. ${ }^{17,29,30}$ This discrepancy could be attributed to the different mechanical properties of these foods. In the case of soft and tough foods such as gels or roast pork, swallowing might be triggered more by factors such as cohesiveness or lubrication than by particle size, and consequently initial particle size and number have little effect on mastication effort. However, in the case of harder foods such as carrots, particle size is a main factor in swallowing determination. ${ }^{24,31}$ Harder foods require smaller bolus particles before the bolus can be swallowed. Consumers therefore use more time and effort to break down all initial particles to a smaller size and to bind these particles together into a cohesive bolus that is safe to swallow. ${ }^{32}$

A comparison of carrot cubes and julienne carrots with similar number of pieces reveals that shape alters the mastication behavior towards carrot pieces to a large extent. Carrot cubes were chewed for shorter time, with fewer chews and with higher eating rate than julienne carrots (Fig. 1). Samples consisting of 8 (C_8), 27 (C_27) and 64 (C_64) carrot cubes were chewed for $21 \pm 1,22 \pm 1$ and $21 \pm 1 \mathrm{~s}$, whereas this was $28 \pm 1$,
$27 \pm 1$ and $25 \pm 1 \mathrm{~s}$ for samples consisting of 8 (J_8), 24 (J_24) and 64 (J_64) julienne pieces, respectively (Fig. 1A). This effect was significant for all sample comparisons; containing 8 pieces $(p<0.001), 27$ versus 24 pieces $(p<0.001)$ and the 64 smallest pieces $(p=0.002)$. The difference in mastication time is also reflected in differences in number of chews and eating rates. C_8, C_27 and C_64 required $28 \pm 1,30 \pm$ and $30 \pm 1$ number of chews and were consumed at eating rates of $12.3 \pm$ $0.4,11.8 \pm 0.5$ and $11.7 \pm 0.3 \mathrm{~g} \mathrm{~min}^{-1}$, whereas this was $38 \pm 2$, $37 \pm 2$ and $34 \pm 2$ chews and $9.3 \pm 0.4,10.0 \pm 0.5$ and $10.5 \pm$ $0.5 \mathrm{~g} \mathrm{~min}{ }^{-1}$ for J_8, J_24 and J_64 (Fig. 1B and C). The number of pieces was not significantly correlated with total mastication time $(r=0.05, p=0.446)$, number of chews $(r=0.04, p=0.501)$ and eating rate $(r=-0.09, p=0.154)$.

These differences in oral processing parameters between the cubes and the julienne carrots are related to the shape of the pieces, and might be explained by differences in aspect ratio or surface area (Table 1). To evaluate how these factors are correlated, Fig. 2 shows mastication time, number of chews and eating rate versus aspect ratio and total surface area of all pieces. Aspect ratio and total surface area of all pieces are both positively correlated with mastication time (aspect ratio: $r=0.37, p<0.001$; surface area: $r=0.42, p<0.001$ ), number of chews (aspect ratio: $r=0.30, p<0.001$; surface area: $r=0.33, p<0.001$ ) and eating rate (aspect ratio: $r=-0.34, p<$ 0.001 ; surface area: $r=-0.41, p<0.001)$. Surface area seems to be stronger correlated with the eating behavior measures than aspect ratio. Aspect ratio and total surface area of all pieces are higher for julienne than for cubes. A higher aspect ratio and surface area would increase the possible contact between the pieces and the teeth, and thereby increase the biting chance. It is easier to bite into a compact cube than into a long thin piece, which often first need to be positioned between the teeth by the tongue before it can be bitten in pieces. However, this does not appear to decrease mastication effort (total mastication time, number of chews). In the case of multiple smaller julienne pieces, they are distributed throughout the oral cavity and more effort is therefore required to place them between the teeth before one can bite. In addition, in the case of a higher surface area, also more saliva (and thus more mastication time) might be needed to paste the julienne particles together to form a bolus that is lubricated and cohesive enough to be safely swallowed. Thus, food particles with a high aspect ratio and/or a high surface area are more difficult to form a safe-to-swallow bolus than those with a low aspect ratio and/or a low surface area. Together, these data highlight that shape has a large impact on mastication effort required to safely swallow raw carrots and consequently also on eating rate. A controlled eating rate could potentially influence vegetable intake. Specific implications of these findings will be discussed later in more detail (section 3.3).

\subsection{Effect of addition of mayonnaises varying in fat content and viscosity on oral processing behavior towards carrots}

To investigate the effect of condiment addition on oral processing behavior, mayonnaises that differed in viscosity and fat 
(A)

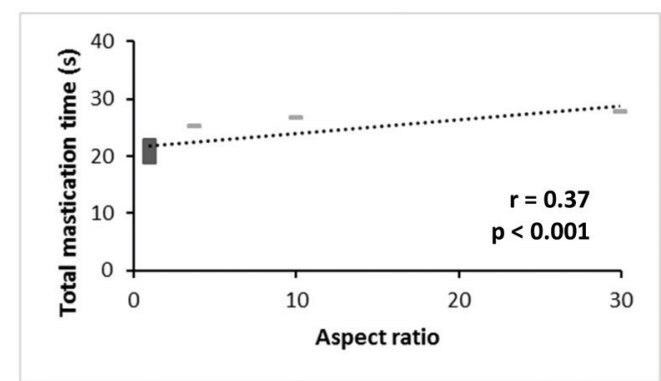

(C)

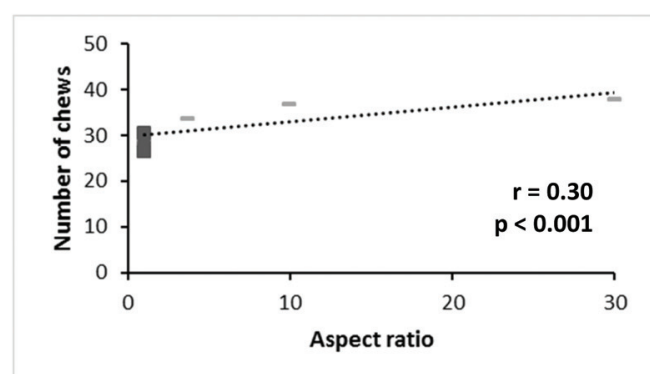

(E)

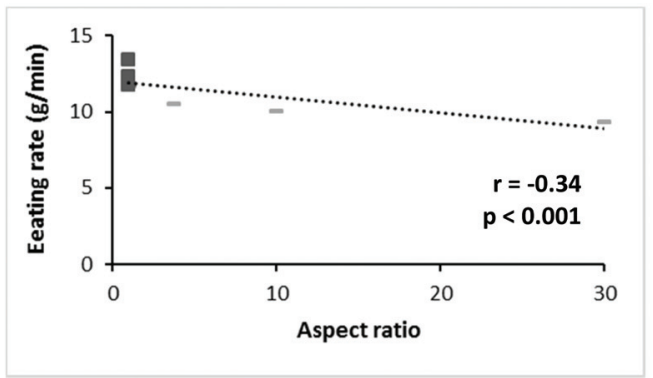

(B)

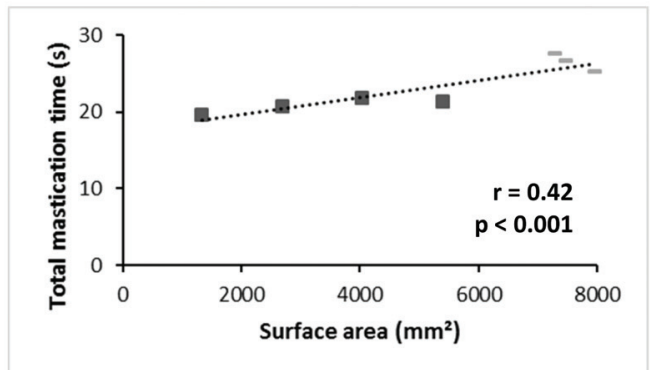

(D)

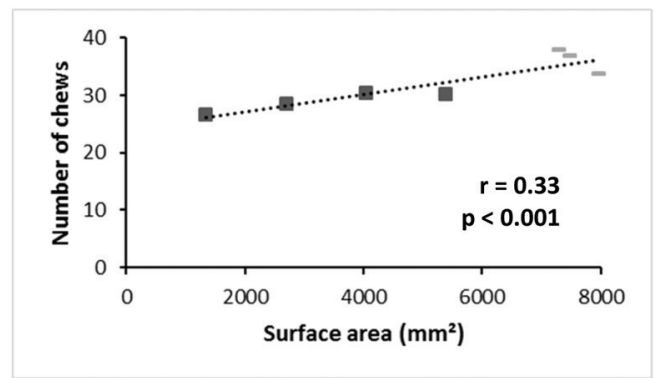

(F)

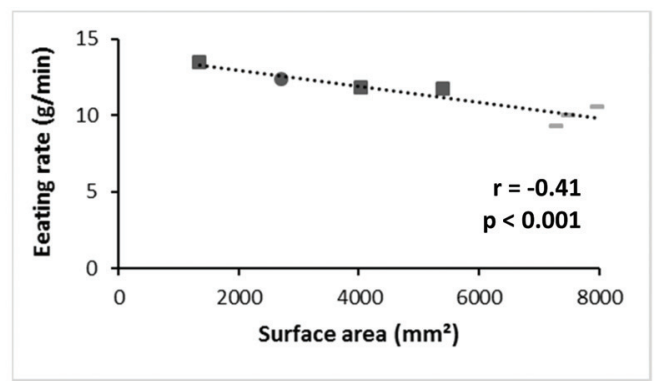

Fig. 2 Correlations among the carrot shape properties aspect ratio (on the left) and total surface area of all pieces (on the right) and the mastication behavior parameters total mastication time (A and B), number of chews (C and D) and eating rate (E and F). The dark-grey squares $(\square)$ represent the cube samples and the light-gray bars $(-$ represent the julienne samples. Pearson's product-moment correlations $(r)$ and $p$-values are shown in the graph.

content were added to carrots (one large cube, C_1 and 8 julienne pieces, J_8). Although mastication effort (total mastication time, number of chews until swallowing, eating rate) of carrots (C_1, J_8) with mayonnaises was mainly driven by the carrot properties, an effect of mayonnaise properties was also observed for mastication time, number of chews and eating rate (mayonnaise effect; $F=10.1, p<0.001$ for total mastication time; $F=5.1, p=0.002$ for number of chews; $F=115.8, p<$ 0.001 for eating rate). Addition of mayonnaise to carrots did not affect chewing frequencies (around 1.3 to 1.4 chews per $\mathrm{s}$ ) of carrot cubes and carrots julienne $(F=1.4, p=0.257)$. As shown in Fig. 3, addition of mayonnaise reduced total mastication time, number of chews until swallowing and increased eating rate of carrots. Although the effects were larger for carrots julienne than for carrot cubes (shape effect; $F=262.3$, $p<0.001$ for total mastication time; $F=133.3, p<0.001$ for number of chews; $F=203.5, p<0.001$ for eating rate), mayonnaise properties (fat content, viscosity) influenced mastication behavior towards C_1 and J_8 carrots in a similar way (no mayonnaise : shape interactions; $F=1.4, p=0.257$ for total mastication time; $F=0.9, p=0.439$ for number of chews; $F=$ $0.7, p=0.576$ for eating rate). When comparing mayonnaises varying in fat content and viscosity, a significant decrease in total mastication time of carrots was observed for full fat (FF_HV) and low viscosity (LF_LV) mayonnaise $(p<0.001, p<$ 0.001 ), but not for low fat high viscosity (LF_HV) mayonnaise 
(A)

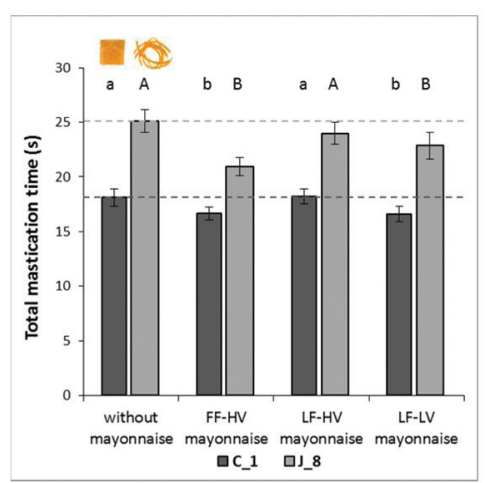

(B)

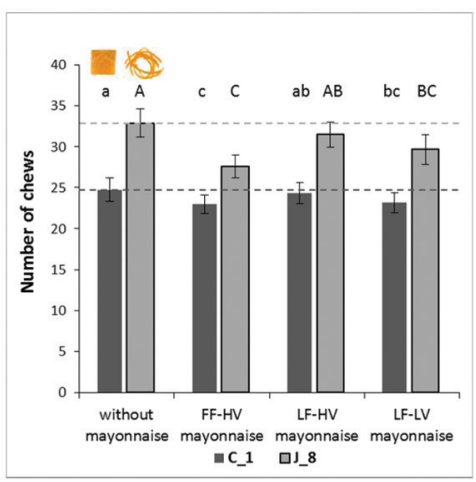

(C)

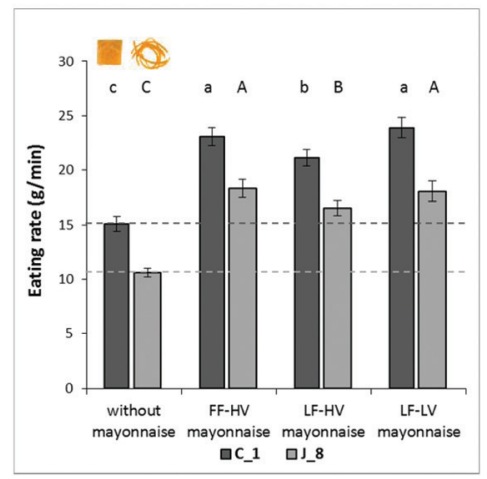

Fig. 3 Total mastication time (A), number of chews until swallowing (B) and eating rate (C) for carrot cube C_1 (dark grey) and carrots julienne $C_{-} 8$ (light grey) without mayonnaise and with different mayonnaises (full fat/high viscosity (FF_HV), low fat/high viscosity (LF_HV), low fat/low viscosity $\left(\mathrm{LF} \_\mathrm{LV}\right)$ ). Carrots alone were served at approximately $4.2 \mathrm{~g}$, and carrots with mayonnaise were served at approximately $6.2 \mathrm{~g}$ (i.e. $4.2 \mathrm{~g}$ carrot, and $2 \mathrm{~g}$ mayonnaise). Error bars represent standard error of the mean. Dashed lines represent mean values of carrots without mayonnaise ( $n=20$ subjects, in duplicate). Different lower case letters indicate significant differences between cube (C_1) without or with different mayonnaises $(p<0.05)$. Different capital letters indicate significant differences between julienne (J_8) without or with different mayonnaises $(p<0.05)$.

$(p=0.489)$. For example, total mastication time of $\mathrm{J} \_8$ decreased from $25 \pm 1$ to $21 \pm 1,24 \pm 1$ and $23 \pm 1 \mathrm{~s}$ after addition of FF_HV, LF_HV and LF_LV mayonnaise, respectively. This difference in mastication time is also reflected in differences in number of chews and eating rates (Fig. 3B and C). Our results show that mayonnaises can aid mastication of carrots and increase eating rate, and that this effect is not driven by mayonnaise fat content alone nor viscosity alone.

When comparing the high viscosity mayonnaises varying in fat content (FF_HV and LF_HV), the results indicate that full fat mayonnaise aids mastication of carrots more than low fat mayonnaise. It could be argued that oil increased lubrication or that oil adhered the carrot fragments more efficiently together than water, and subsequently triggered earlier swallowing. Another possible explanation might be that the presence of a relative high amount of xanthan in the LF_HV mayonnaise slowed down bolus formation. Although the mayonnaise had a similar viscosity profile as the FF-HV mayonnaise (Table 2), the LF_HV mayonnaise had a gummy structure, possibly causing slower adherence of the mayonnaise to the carrot particles. As a consequence, a higher effort was required to adhere carrot particles together compared to that of the full fat version, by which more time was needed to form a bolus that is safe to swallow. When comparing the low fat mayonnaises varying in viscosity (LF_HV and $\mathrm{LF}_{-} L \mathrm{LV}$ ), a low viscous mayonnaise shortened mastication of carrots more than the high viscous mayonnaise. This could be attributed to faster carrot particle adherence due to the lower viscosity, and consequently triggered swallowing after shorter mastication times. Hence, mayonnaises with a high fat content or a low viscosity adhered to and lubricated carrot particles better, leading to shorter mastication times to form a bolus that is safe enough to be swallowed. Specific implications of these findings will be discussed later in more detail (section 3.3).
The condiments seem to influence the eating behavior by affecting the bolus formation. To gain more insights into this effect, the carrot bolus properties (particle size, particle retention) at the moment of swallowing were determined (Fig. 4, Table 3). In case of carrots without mayonnaise, $0.5 \mathrm{~g}$ bolus of a carrot cube (C_1) consisted of $74 \pm 4$ particles with a $d_{50}$ of $4.8 \pm 0.3$, whereas carrot julienne (J_8) consisted of $155 \pm 6$ particles with a $d_{50}$ of $3.9 \pm 0.2 \mathrm{~mm}^{2}$. The presence of mayonnaises clearly influenced carrot particle size distributions of both carrot cubes and carrots julienne (Fig. 4, Table 3). Carrots were broken down into a bolus consisting of fewer particles of larger sizes when any of the mayonnaises were added. On average, the influence of mayonnaise on the bolus properties of carrots was larger for carrots julienne (J_8) than carrot cubes (C_1) (mayonnaise : shape interaction effects; $F=14.2, p$ $<0.001$ for particle count; $F=7.0, p<0.001$ for particle size; $F$ $=3.3, p=0.024$ for carrot retention in bolus). In the case of carrots julienne (J_8) with $\mathrm{FF} \_$HV, LF_HV and LF_LV mayonnaise, $0.5 \mathrm{~g}$ of bolus consisted of $117 \pm 6,97 \pm 4$ and $114 \pm 4$ particles with a $d_{50}$ of $5.9 \pm 0.3,7.5 \pm 0.4$ and $5.8 \pm 0.3 \mathrm{~mm}^{2}$, respectively, which are fewer particles of much larger sizes than in the bolus without mayonnaise $(155 \pm 6$ particles with a $d_{50}$ of $3.9 \pm 0.2 \mathrm{~mm}^{2}$ ). In addition, the presence of mayonnaise increased the mass of carrot retained in the bolus for carrots julienne (J_8) (Table 3). Consequently, addition of mayonnaise decreased the mass of carrot lost ( $\left.m_{\text {lost }}\right)$ during chewing carrots julienne. These results show that mayonnaises assist saliva in bolus formation of carrots. This is consistent with our previous hypothesis that mayonnaises increase bolus cohesiveness by adhering carrot pieces together and at the same time provide lubrication. Due to the larger surface area of the julienne, more particles were adhered by the mayonnaise and consequently included in the bolus. Due to the incorporation of the mayonnaise into the bolus, the carrot boli can be safely 

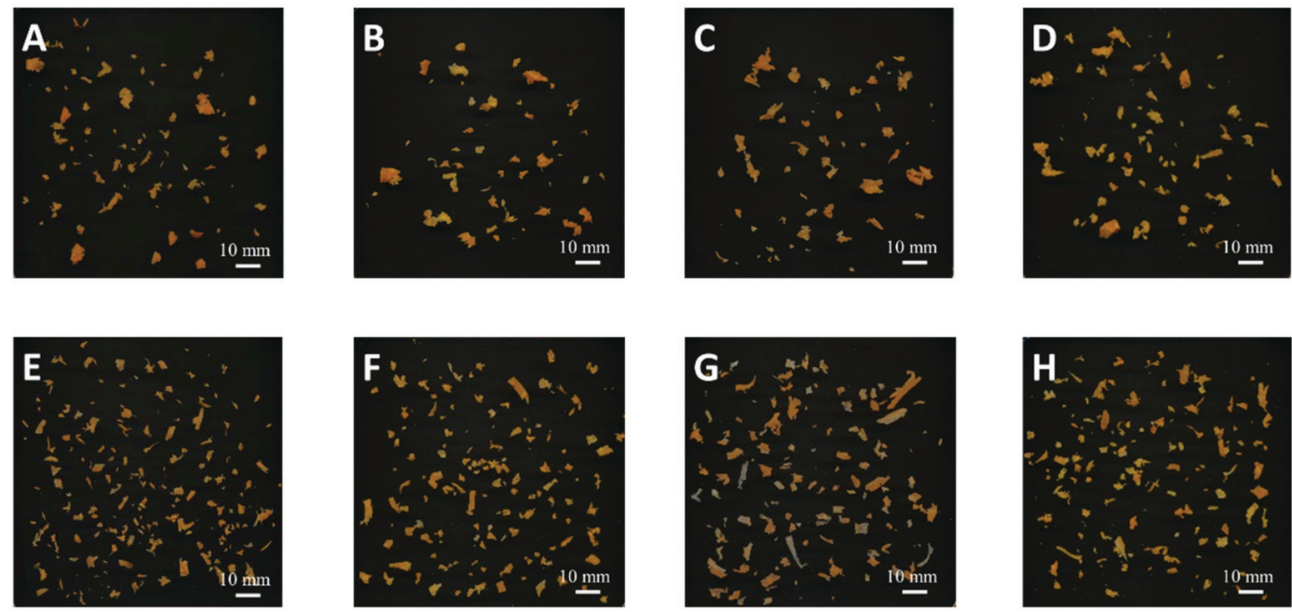

Fig. 4 Pictures of expectorated carrot fragments $(0.5 \mathrm{~g})$ at the moment of swallowing of one subject (A-D: carrot cube C_1 without mayonnaise, and with full fat/high viscosity (FF_HV), low fat/high viscosity (LF_HV) and low fat/low viscosity (LF_LV) mayonnaise; E-H: carrots julienne J_8 without mayonnaise, and with full fat/high viscosity (FF_HV), low fat/high viscosity (LF_HV) and low fat/low viscosity (LF_LV) mayonnaise).

swallowed at larger particle sizes after shorter eating times. Although condiments are known to facilitate bolus formation of dry, low moisture content foods such as bread or crackers, ${ }^{20-22}$ this is the first time that condiments are shown to assist bolus formation of foods with a high moisture content without moisture absorbing capacity. Hence, the mechanism by which condiments facilitate bolus formation of carrier foods depends on the properties of the carrier foods. In the case of dry bread and crackers, the condiments provide moistening and softening. In the case of carrots, condiments are used to provide increased bolus cohesiveness. Providing lubrication seems to play a role in both dry and moist carrier foods and is therefore likely to be an important parameter in safe swallowing.

Mayonnaises varying in fat content and viscosity impacted the mastication behavior towards carrots differently. FF_HV and LF_LV mayonnaises significantly decreased the mastication effort (total mastication time, number of chews) required for the consumption of carrot cubes and carrots julienne (Fig. 3), whereas little effects were observed for LF_HV mayonnaise. Although the LF_HV mayonnaise had the lowest impact on mastication behavior, it largely influenced carrot bolus properties including carrot particle size and carrot retention in the bolus (Table 3). Addition of the LF_HV mayonnaise decreased the number of particles, increased the size of the particles and increased carrot retention in the bolus to a larger extent than the FF_HV and LF_LV mayonnaises. This shows that when carrots are consumed in combination with mayonnaise, it is not the particle size that determines the swallowing threshold. ${ }^{33}$ Instead other bolus properties such as lubrication or cohesiveness seem to dominate the swallowing threshold and trigger the urge to swallow.

As summarized in the Principal Component Analysis (PCA) bi-plot (Fig. 5), mastication behavior and bolus properties of carrots are mainly affected by carrot shape (PC1, 72.4\%). Carrots julienne were chewed for a longer time with more chews to decrease the degree of structure (i.e. higher number of particles). As a consequence of this longer chewing time, less carrot was retained in the bolus likely due to intermediate swallows. However, when mayonnaise was added, the amount of carrots in the bolus was increased more for the julienne than for the cubes. The type of mayonnaise induced some smaller changes to the mastication behavior and bolus properties of carrots (PC2, 23.8\%). 95\%-confidence ellipses indicate that julienne without mayonnaise (J_8) differed significantly from all julienne-mayonnaise combinations, whereas cube without mayonnaise (C_1) differed significantly from cube with LF_HV mayonnaise only, and not from the FF_HV and LF_LV mayonnaise. These results indicate that mayonnaise had a larger impact on bolus formation of julienne carrots than for carrot cubes.

\subsection{Practical implications}

Practical approaches to increase vegetable consumption are desired, as many people do not meet the recommended daily intake of vegetables. Results showed that changing the shape of carrots affected mastication time (Fig. 1A) and eating rate (Fig. 1C), which are known to influence food intake within a meal. ${ }^{7,10-12}$ We observed that carrot cubes were consumed with less mastication effort (shorter mastication time, fewer chews) and with higher eating rates than julienne carrots. Furthermore, carrots cut into one large cube were consumed with lower mastication effort and higher eating rate than for small carrots already cut into smaller pieces. Hence, cutting vegetables in particular into elongated, julienne pieces (high aspect ratio, high surface area) prolongs mastication time, and slows down eating rate. This would potentially reduce vegetable intake. On the other hand, consuming vegetables in larger pieces may increase vegetable intake. Thus, vegetable shape influences eating rate and potentially influences vegetable intake. It has to be noted that changing vegetable properties within one bite (standardized eating) can be different 


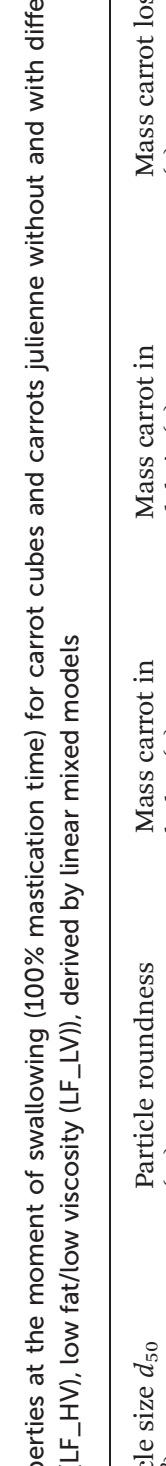



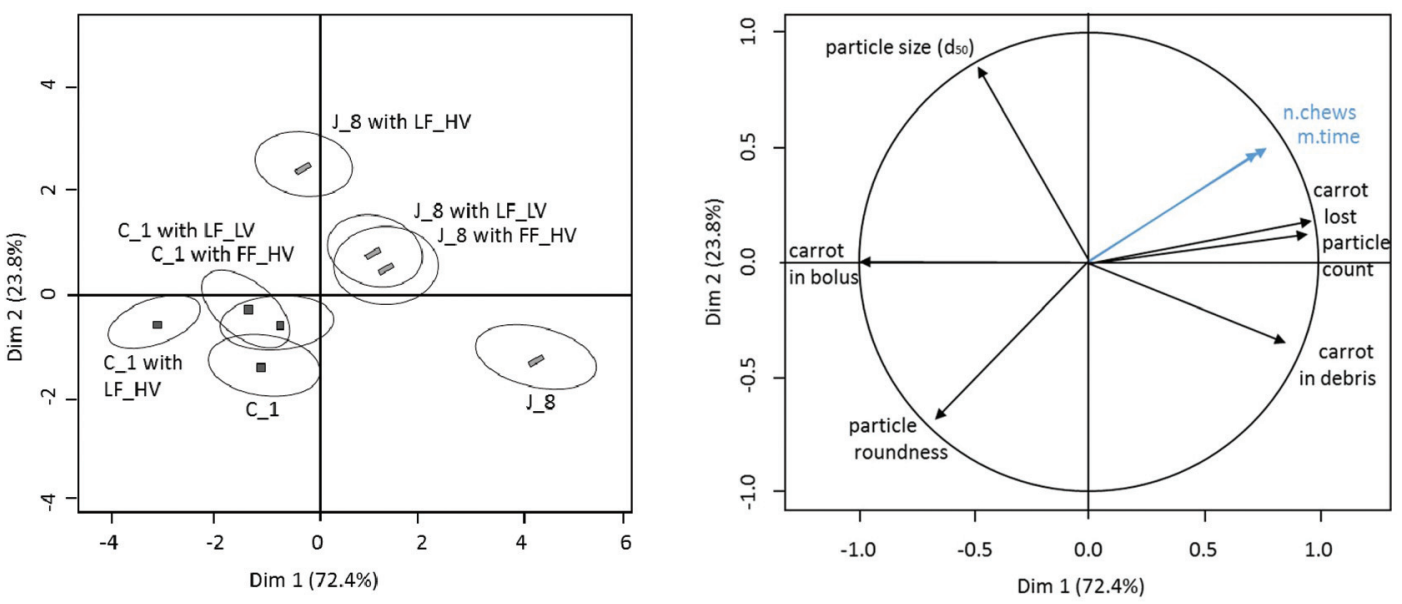

Fig. 5 Principal component analysis (PCA) of the bolus parameters of carrot cubes $\left(C \_1, \square\right)$ and carrots julienne $\left(J_{-} 8, \square\right)$ without mayonnaise and with different mayonnaises (full fat/high viscosity (FF_HV), low fat/high viscosity (LF_HV), low fat/low viscosity (LF_LV)) at $100 \%$ of total mastication time. Mastication behavior parameters are plotted as supplementary variables (blue lines). The individuals map (samples) is shown on the left, and the variables map (attributes) is shown on the right. The ellipse level of confidence was 0.95 .

it can even increase mastication effort. As discussed above, adding condiments to solid foods, in particular those condiments with a low viscosity or a high fat content, aids chewing and is expected to facilitate increased food intake of older consumers with decreased eating capabilities.

Next to food intake, nutrient absorption from vegetables into the human body is important to assure the beneficial impact of nutrients on human health. As discussed before, initial carrot shape, and the presence and type of mayonnaise affect carrot particle sizes at the moment of swallowing (Fig. 4, Table 3). We speculate that this might have important implications for the bioaccessibility of micronutrients present in carrots, such as $\alpha$-carotene, $\beta$-carotene, vitamin $\mathrm{K}$ and vitamin B6. For raw carrots, it has been shown that carotene bioaccessibility increases when carrot particle sizes decrease at the moment of swallowing. ${ }^{37,38}$ We observed that carrot cubes (C_1) were swallowed with larger particle sizes than carrots cut into long julienne pieces (J_8). To facilitate micronutrient absorption, we would therefore recommend to cut vegetables before consumption, leading to longer mastication times and consequently further particle breakdown before swallowing. This recommendation is in contrast to our recommendation on food intake. From an increased bioaccesibility perspective we recommend to pre-cut vegetables, whereas from a decreased ad libitum intake perspective we recommend to eat whole/large vegetables. To develop a full picture of vegetable intake and subsequent nutrient absorption, additional multidisciplinary studies are needed that combine eating behavior, bolus properties and digestion of vegetables. Further, the present study showed that addition of mayonnaises increased carrot particle sizes at the moment of swallowing, and thus a decrease in carotene bioaccessibility can be expected. On the other hand, the presence of lipids is known to facilitate carotenoid accessibility. ${ }^{39}$ Brown et al. found a greater absorption of carotenoids when raw carrots were consumed in combi- nation with salad dressings containing fat. ${ }^{40}$ Thus, although larger carrot pieces were swallowed with the presence of mayonnaises, this lower accessibility might be compensated by a higher uptake due to the presence fat. This holds in particular for the full fat mayonnaise (FF_HV). The combination of these findings provides some support that the addition of condiments to carrots, regardless of the particle size, can increase carotenoid accessibility.

\section{Conclusions}

This study highlights that carrot shape has a large impact on mastication effort required to safely swallow carrots. Carrots cut in one large cube required the shortest chewing time and the least chews among all pre-cut carrots (cubes and julienne). Carrot cubes were chewed for a shorter time, with fewer chews and with higher eating rate than carrots julienne with similar number of pieces and an equal total weight. Aspect ratio and surface area increase with pre-cutting and are higher for julienne than for cube carrots. Consequently, more chewing and more saliva is needed to break down and pack the carrot particles together to form a lubricated and cohesive bolus that is safe to swallow. Furthermore, addition of mayonnaise reduced total mastication time until swallowing and increased eating rate of both carrot cubes and carrots julienne. Carrots were swallowed with less particles of larger sizes when mayonnaises were added. A specific particle size is therefore not a prerequisite to induce swallowing. This indicates that other bolus properties such as lubrication or cohesiveness rather than carrot particle size triggered the urge to swallow. In particular, full fat mayonnaise and low viscous mayonnaise lubricated and adhered carrot particles more than low fat high viscosity mayonnaise, leading to shorter mastication times and a lower number of chewing cycles to form boli that can be safely swal- 
lowed. We conclude that oral processing behavior towards carrot-mayonnaise combinations is mainly affected by carrot shape and to a smaller extent by the presence and type of mayonnaise. These results suggest that relatively small changes in the vegetable or condiment properties can largely alter consumption time and eating rate, which could be an effective strategy to increase vegetable consumption or to decrease mastication effort to target the elderly population.

\section{Conflicts of interest}

There are no conflicts to declare.

\section{Acknowledgements}

The project is funded by TiFN, a public - private partnership on precompetitive research in food and nutrition. The public partners are responsible for the study design, data collection and analysis, decision to publish, and preparation of the manuscript. The private partners have contributed to the project through regular discussion. The private partners are Royal Friesland Campina, Fromageries Bel and Unilever. This research was performed with additional funding from the Top Consortia for Knowledge and Innovation of the Dutch Ministry of Economic Affairs.

\section{References}

1 J. L. Slavin and B. Lloyd, Health benefits of fruits and vegetables, Adv. Nutr., 2012, 3, 506-516.

2 X. Wang, Y. Ouyang, J. Liu, M. Zhu, G. Zhao, W. Bao and F. B. Hu, Fruit and vegetable consumption and mortality from all causes, cardiovascular disease, and cancer: systematic review and dose-response meta-analysis of prospective cohort studies, Br. Med. J., 2014, 349, g4490.

3 K. Diethelm, N. Jankovic, L. A. Moreno, I. Huybrechts, S. De Henauw, T. De Vriendt, M. Gonzalez-Gross, C. Leclercq, F. Gottrand, C. C. Gilbert, J. Dallongeville, M. Cuenca-Garcia, Y. Manios, A. Kafatos, M. Plada and M. Kersting, Food intake of European adolescents in the light of different food-based dietary guidelines: results of the HELENA (Healthy Lifestyle in Europe by Nutrition in Adolescence) Study, Public Health Nutr., 2012, 15, 386-398.

4 C. Vereecken, T. P. Pedersen, K. Ojala, R. Krolner, A. Dzielska, N. Ahluwalia, M. Giacchi and C. Kelly, Fruit and vegetable consumption trends among adolescents from 2002 to 2010 in 33 countries, Eur. J. Public Health, 2015, 25(Suppl. 2), 16-19.

5 C. Van Rossum, E. Buurma, F. Vennemann, M. Beukers, J. Drijvers and M. Ocké, Voedselconsumptie in 2012-2014 vergeleken met de Richtlijnen goede voeding 2015, 2017.

6 C. Van Rossum, E. Buurma-Rethans, F. Vennemann, M. Beukers, H. A. Brants, E. De Boer and M. C. Ocké, The diet of the Dutch: Results of the first two years of the
Dutch National Food Consumption Survey 2012-2016, RIVM letter report 2016-0082, 2016.

7 E. Robinson, E. Almiron-Roig, F. Rutters, C. de Graaf, C. G. Forde, C. Tudur Smith, S. J. Nolan and S. A. Jebb, A systematic review and meta-analysis examining the effect of eating rate on energy intake and hunger, Am. J. Clin. Nutr., 2014, 100, 123-151.

8 M. Devezeaux de Lavergne, J. A. M. Derks, E. C. Ketel, R. A. de Wijk and M. Stieger, Eating behaviour explains differences between individuals in dynamic texture perception of sausages, Food Quality Preference, 2015, 41, 189-200.

9 E. C. Ketel, M. G. Aguayo-Mendoza, R. A. de Wijk, C. de Graaf, B. Piqueras-Fiszman and M. Stieger, Age, gender, ethnicity and eating capability influence oral processing behaviour of liquid, semi-solid and solid foods differently, Food Res. Int., 2019, 119, 143-151.

10 C. G. Forde, C. Leong, E. Chia-Ming and K. McCrickerd, Fast or slow-foods? Describing natural variations in oral processing characteristics across a wide range of Asian foods, Food Funct., 2017, 8(2), 595-606.

11 C. G. Forde, N. van Kuijk, T. Thaler, C. de Graaf and N. Martin, Oral processing characteristics of solid savoury meal components, and relationship with food composition, sensory attributes and expected satiation, Appetite, 2013, 60, 208-219.

12 J. van den Boer, M. Werts, E. Siebelink, C. de Graaf and M. Mars, The availability of slow and fast calories in the Dutch diet: The current situation and opportunities for interventions, Foods, 2017, 6, 87.

13 M. G. Aguayo-Mendoza, E. C. Ketel, E. van der Linden, C. G. Forde, B. Piqueras-Fiszman and M. Stieger, Oral processing behavior of drinkable, spoonable and chewable foods is primarily determined by rheological and mechanical food properties, Food Quality Preference, 2019, 71, 87-95.

14 D. P. Bolhuis, C. G. Forde, Y. Cheng, H. Xu, N. Martin and C. de Graaf, Slow Food: Sustained Impact of Harder Foods on the Reduction in Energy Intake over the Course of the Day, PLoS One, 2014, 9, e93370.

15 C. L. Campbell, T. B. Wagoner and E. A. Foegeding, Designing foods for satiety: The roles of food structure and oral processing in satiation and satiety, Food Struct., 2017, 13, 1-12.

16 M. P. Lasschuijt, M. Mars, M. Stieger, S. Miquel-Kergoat, C. de Graaf and P. A. M. Smeets, Comparison of orosensory exposure duration and intensity manipulations on satiation, Physiol. Behav., 2017, 176, 76-83.

17 K. Kohyama, Y. Nakayama, I. Yamaguchi, M. Yamaguchi, F. Hayakawa and T. Sasaki, Mastication efforts on block and finely cut foods studied by electromyography, Food Quality Preference, 2007, 18, 313-320.

18 J. R. Goh, C. G. Russell and D. G. Liem, An Investigation of Sensory Specific Satiety and Food Size When Children Consume a Whole or Diced Vegetable, Foods, 2017, 6, 55.

19 D. G. Liem and C. G. Russell, Supersize me. Serving carrots whole versus diced influences children's consumption, Food Quality Preference, 2019, 74, 30-37. 
20 L. Engelen, A. Fontijn-Tekamp and A. Van Der Bilt, The influence of product and oral characteristics on swallowing, Arch. Oral Biol., 2005, 50, 739-746.

21 M. B. D. Gavião, L. Engelen and A. Van Der Bilt, Chewing behavior and salivary secretion, Eur. J. Oral Sci., 2004, 112, 19-24.

22 A. van Eck, N. Hardeman, N. Karatza, V. Fogliano, E. Scholten and M. Stieger, Oral processing behavior and dynamic sensory perception of composite foods: Toppings assist saliva in bolus formation, Food Quality Preference, 2019, 71, 497-509.

23 J. Chen, N. Khandelwal, Z. Liu and T. Funami, Influences of food hardness on the particle size distribution of food boluses, Arch. Oral Biol., 2013, 58, 293-298.

24 M. L. Jalabert-Malbos, A. Mishellany-Dutour, A. Woda and M. A. Peyron, Particle size distribution in the food bolus after mastication of natural foods, Food Quality Preference, 2007, 18, 803-812.

25 A. Kuznetsova, P. B. Brockhoff and R. H. B. Christensen, lmerTest: Tests in Linear Mixed Effects Models, 2016.

26 F. Husson, S. Le and M. Cadoret, SensoMineR: Sensory data analysis with R, 2014.

27 P. W. Lucas and D. A. Luke, Methods for analysing the breakdown of food in human mastication, Arch. Oral Biol., 1983, 28, 813-819.

28 H. Van der Glas, A. Van der Bilt, L. Olthoff and F. Bosman, Measurement of selection chances and breakage functions during chewing in man, J. Dent. Res., 1987, 66, 1547-1550.

29 E. H. J. Kim, V. B. Jakobsen, A. J. Wilson, I. R. Waters, L. Motoi, D. I. Hedderley and M. P. Morgenstern, Oral Processing of Mixtures of Food Particles, J. Texture Stud., 2015, 46, 487-498.

30 K. Kohyama, Y. Nakayama, H. Watanabe and T. Sasaki, Electromyography of Eating Apples: Influences of Cooking, Cutting, and Peeling, J. Food Sci., 2005, 70, S257-S261.
31 M. A. Peyron, A. Mishellany and A. Woda, Particle size distribution of food boluses after mastication of six natural foods, J. Dent. Res., 2004, 83, 578-582.

32 K. Nishinari, M. Turcanu, M. Nakauma and Y. Fang, Role of fluid cohesiveness in safe swallowing, npj Sci. Food, 2019, 3, 5.

33 J. B. Hutchings and P. J. Lillford, The perception of food texture - The philosophy of the breakdown path, J. Texture Stud., 1988, 19, 103-115.

34 M. Hickson, Malnutrition and ageing, Postgrad. Med. J., 2006, 82, 2-8.

35 L. Pauly, P. Stehle and D. Volkert, Nutritional situation of elderly nursing home residents, Z. Gerontol., 2007, 40, 312.

36 L. Mioche, P. Bourdiol, S. Monier, J. F. Martin and D. Cormier, Changes in jaw muscles activity with age: Effects on food bolus properties, Physiol. Behav., 2004, 82, 621-627.

37 E. Hedrén, V. Diaz and U. Svanberg, Estimation of carotenoid accessibility from carrots determined by an in vitro digestion method, Eur. J. Clin. Nutr., 2002, 56, 425.

38 L. Lemmens, S. Van Buggenhout, A. M. Van Loey and M. E. Hendrickx, Particle Size Reduction Leading to Cell Wall Rupture Is More Important for the $\beta$-Carotene Bioaccessibility of Raw Compared to Thermally Processed Carrots, J. Agric. Food Chem., 2010, 58, 12769-12776.

39 A. A. O. Xavier and A. Z. Mercadante, The bioaccessibility of carotenoids impacts the design of functional foods, Curr. Opin. Food Sci., 2019, DOI: 10.1016/j.cofs.2019.02.015.

40 M. J. Brown, M. G. Ferruzzi, M. L. Nguyen, D. A. Cooper, A. L. Eldridge, S. J. Schwartz and W. S. White, Carotenoid bioavailability is higher from salads ingested with full-fat than with fat-reduced salad dressings as measured with electrochemical detection, Am. J. Clin. Nutr., 2004, 80, 396403. 